\title{
In-medium Hadrons - Properties, Interaction and Formation
}

\author{
Kai Gallmeister ${ }^{\mathrm{a}}$, Tina Leitner ${ }^{\mathrm{a}}$, Stefan Leupold ${ }^{\mathrm{a}}$, Ulrich Mosel $^{\mathrm{a} *}$, Pascal Muehlich $^{\mathrm{a}}$, Luis \\ Alvarez-Ruso $^{\text {a }}$, Vitaly Shklyar ${ }^{\text {a }}$ \\ ${ }^{a}$ Institut fuer Theoretische Physik, Universitaet Giessen, D-35392 Giessen, Germany
}

In this talk various aspects of in-medium behavior of hadrons are discussed with an emphasis on observable effects. Examples for theoretical predictions of in-medium spectral functions are given and the importance of resonance-hole excitations is stressed. It is also stressed that final state interactions can have a major effect on observables and thus have to be considered as part of the theory. This is demonstrated with examples from neutrino-nucleus interactions. Finally, the possibility to access hadron formation times in high-energy photonuclear (or neutrino-induced) reactions is illustrated.

\section{Introduction}

Hadrons, embedded inside nuclei, obviously change some of their properties. They acquire complex selfenergies with the real parts reflecting the binding (or non-binding) properties and the imaginary parts reflecting the interactions and possibly their changes inside the medium. Particles that are produced through resonances or - at high energies - through strings become physical, on-shell particles only after some formation time. In this case the nuclear medium may affect the formation process and can thus act as a micro-detector for the early stages of particle production.

Naively, one expects that in lowest order all in-medium effects go linearly with the density of nuclear matter, $\rho$, around the hadron. This has triggered a series of experiments with relativistic and ultrarelativistic heavy-ions, which can reach high densities, that have looked for such effects and have indeed reported in-medium changes of the $\rho$ meson [1, 2, 3]. However, it has been pointed out quite early [4] that also experiments with microscopic probes on nuclei can yield in-medium signals that are as large as those obtained in heavyion collisions. Although, of course, the density probed here is always below $\rho_{0}$ the observed signal is cleaner in the sense that it does not contain an implicit integration over very different phases of the reaction and the nuclear environment. The signal to be expected is also nearly as large as that seen in heavy-ion collisions. This idea has been followed up in recent experiments with photons on nuclei [ [5, 6], where indeed changes of the $\omega$ meson in medium have been reported [ [6].

In this talk I will give an overview of this field with an emphasis on observable effects. I will also stress the need for a theoretical framework that allows to follow the signals from

*e-mail: mosel@physik.uni-giessen.de

${ }^{\dagger}$ Work supported by BMBF and DFG 
the hadron inside nuclei all the way to the final detector. More details can be found in two previous reviews [ [7, 8].

\section{Vector mesons in medium}

The original suggestion by Hatsuda and Lee [9] that QCD sum rules predict a significant lowering of the vector meson masses in medium already at saturation density has created quite an excitement at that time. We now understand that this prediction was overly simplistic [10, 11] and that it has to be replaced by the prediction that spectral strength of the vector mesons moves down to lower invariant masses. The latter can be achieved not only by a downwards mass-shift, but also by a broadening, or a multi-humped structure, of the vector meson spectral function. QCD sum rules thus do not predict specifics of inmedium hadron spectral functions, but they do provide constraints for model predictions. They also link - although very indirectly - the spectral functions seen in the hadronic world to the quark- and gluon-condensates in the QCD world.

Thus, for a specific prediction of the in-medium spectral function a hadronic model is necessary. It has been realized by now that in particular resonance-hole contributions have a major influence on hadronic in-medium properties. For the pion this has been known for a long time; the Delta-hole model explains a large part of the observed pionnucleus interactions [12]. As in this particular case the most essential ingredient is the coupling constant of the resonance-nucleon-meson vertex, which is obviously large in the Delta-nucleon-pion case. Thus, at this point nucleon resonance physics and in-medium physics meet!

For the $\rho$ meson the most essential resonance is the $D_{13}(1520)$ excitation which according to the PDG - has a partial decay width into $\rho N$ of about $20 \%$. This directly translates into a large coupling constant since this resonance lies about $200 \mathrm{MeV}$ below the nominal threshold for $\rho N$ decay if the decay width given is indeed correct $^{3}$.

The result of a calculation of the transverse part of the $\rho$ spectral function employing the 'official' coupling strength is shown in Fig. 1.

The calculated spectral function shows significant structure that directly reflects various nucleon resonances, the most dominant one being indeed the $N(1520)$ resonance which leads to the sharp second peak at invariant masses around $1500 \mathrm{MeV}$. One also notices that there is a strong momentum-dependence in the spectral function and that in particular this low-mass hump disappears at higher momenta where the spectral function still shows considerable broadening compared to the free case, but no longer the mass-shift seen at small momenta [14].

\subsection{Omega Meson}

Exactly the same model has been applied recently to the in-medium selfenergy of the $\omega$ meson. Early models for this meson had mainly invoked a change of the pion cloud in medium; such studies were restricted to tree-level diagrams with very limited basis space (no resonances to dress the mesons involved) [15].

\footnotetext{
${ }^{3}$ This 'subthreshold decay' has never explicitly been seen experimentally, but has been inferred from an analysis of pion inelasticities. A direct verification - possible by a partial wave analysis of $2 \pi$ channels could help to clarify this essential point.
} 


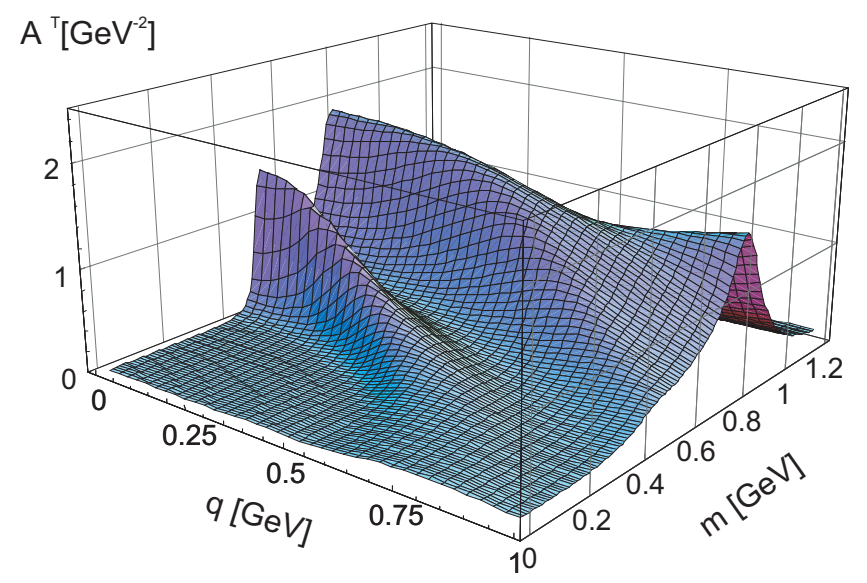

Figure 1. Selfconsistent (to all orders of density $\rho$ ) spectral function of transverse $\rho$ mesons in nuclear matter at equilibrium density $\rho_{0}$ (from [13]).

A complication when applying the resonance-hole model also to the $\omega$ meson lies in the fact that here the experimental knowledge on the resonance-nucleon-omega coupling is still much more uncertain than for the $\rho$ meson. Until a few years ago, no resonances were known that decay into $N \omega$ and only recently experiments [16] and analyses [17, 18, 19] have become available. The results on the coupling still change from analysis to analysis; it is clear, however, that background terms play a large role here in addition to resonance couplings.

The results of a very recent analysis are shown in Fig. 2. The calculations use - in lowest order in the density - the results of a simultaneous coupled channel analysis of $(\gamma N)$ and $(\pi N)$ reactions [19] and in this way goes far beyond earlier analyses which either contained no resonance-coupling at all [15] or invoked VMD to relate the photon-nucleon coupling to the $\omega N$ one [18, 21]. The $\omega$ spectral function at normal nuclear density is considerably broadened, but hardly shows a shift at all (see Fig. 2). This is due to the comparatively small coupling to resonance-hole states that, in addition, leads to a visible $N^{*}$ hole component $\left(S_{11}(1535)\right)$ only far below the free $\omega$ mass.

As for the $\rho$ meson the transverse and the longitudinal components behave quite differently in medium. Whereas the longitudinal component changes its shape only very little the transverse component gets significantly suppressed and broadened.

Recently, the CBELSA/TAPS experiment has reported evidence for a change of the omega-in-medium spectral properties and has found a lowering of the omega signal [ 6]. BUU calculations that take the rescattering of the pion in the outgoing channel into account can reproduce this signal when a lowering of the $\omega$ mass by about $14 \%$ at $\rho_{0}$ is put in by hand [22]. The width obtained in the same experiment of about $55 \mathrm{MeV}$ for slow $\omega_{\mathrm{s}}$ is of the same order as that predicted theoretically [20], but is still determined by experimental resolution. The observed downward shift of strength is contrary to what the calculation mentioned above predicts. However, the experimental result involves a product of spectral function and partial decay width into the $\pi^{0} \gamma$ channel that was used in the experiment and it is this product that shows the downward trend. How the spectral 


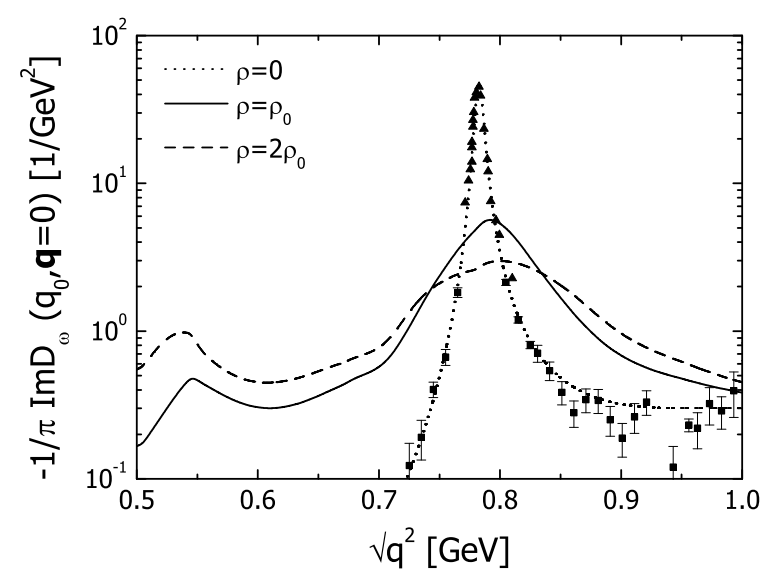

Figure 2. The spectral function for an $\omega$ meson at rest. The appropriately normalized data points correspond to the reaction $e^{+} e^{-} \rightarrow \omega \rightarrow 3 \pi$ in vacuum. Shown are results for densities $\rho=0, \rho=\rho_{0}=0.16 \mathrm{fm}^{3}$ (solid) and $\rho=2 \rho_{0}$ (dashed)(from [20]).

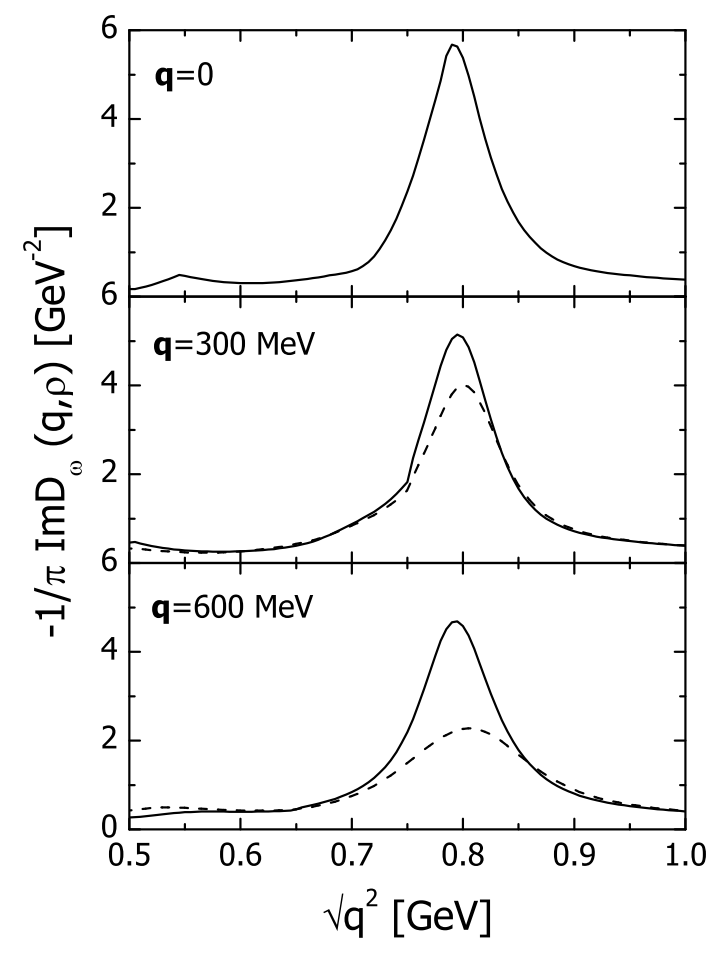

Figure 3. The longitudinal (solid) and transverse (dashed) spectral functions for an $\omega$ meson at various momenta given in the figure (from [20]. 
function by itself behaves in medium must be the subject of further studies.

\section{Neutrino Reactions}

A very dramatic example for the action of final state interactions is provided by the charged current neutrino-induced neutron knockout off nuclei. Since charged current (CC) interactions by themselves always change the charge of the hit nucleon by one unit there cannot be any CC knock-out neutrons in a quasielastic process. This is indeed born out in the results of calculations (see Fig. 4, left) [23]. The few events visible in that picture at $Q^{2} \approx 0.05 \mathrm{GeV}^{2}$ and $E_{\mu} \approx 0.6 \mathrm{GeV}$ stem from events where first a $\Delta^{+}$is produced that then decays into $\pi^{+} n$.

Final state interactions (FSI) are now being implemented by means of the GiBUU transport method [ 24]. When these final state interactions, that involve elastic and inelastic rescattering, particle production and sidefeeding, are turned on this picture changes dramatically (see Fig. 4, right). Now a significant neutron knockout signal appears at $E_{\mu} \approx 0.9 \mathrm{GeV}$ with a long ridge in $Q^{2}$. In addition the $\Delta$-like events now show also considerably more strength. The former effect is caused by charge-transfer reactions where in a first interaction a proton is knocked on that then travels through the nucleus and transmits its energy and momentum to a hit neutron that is being knocked out of the nucleus. The same applies to the $\Delta$-like events: due to charge-exchange FSI now also the initial decay channels $\Delta^{+} \rightarrow \pi^{0} p$ and $\Delta^{++} \rightarrow \pi^{+} p$ can contribute to final neutrons being knocked out.
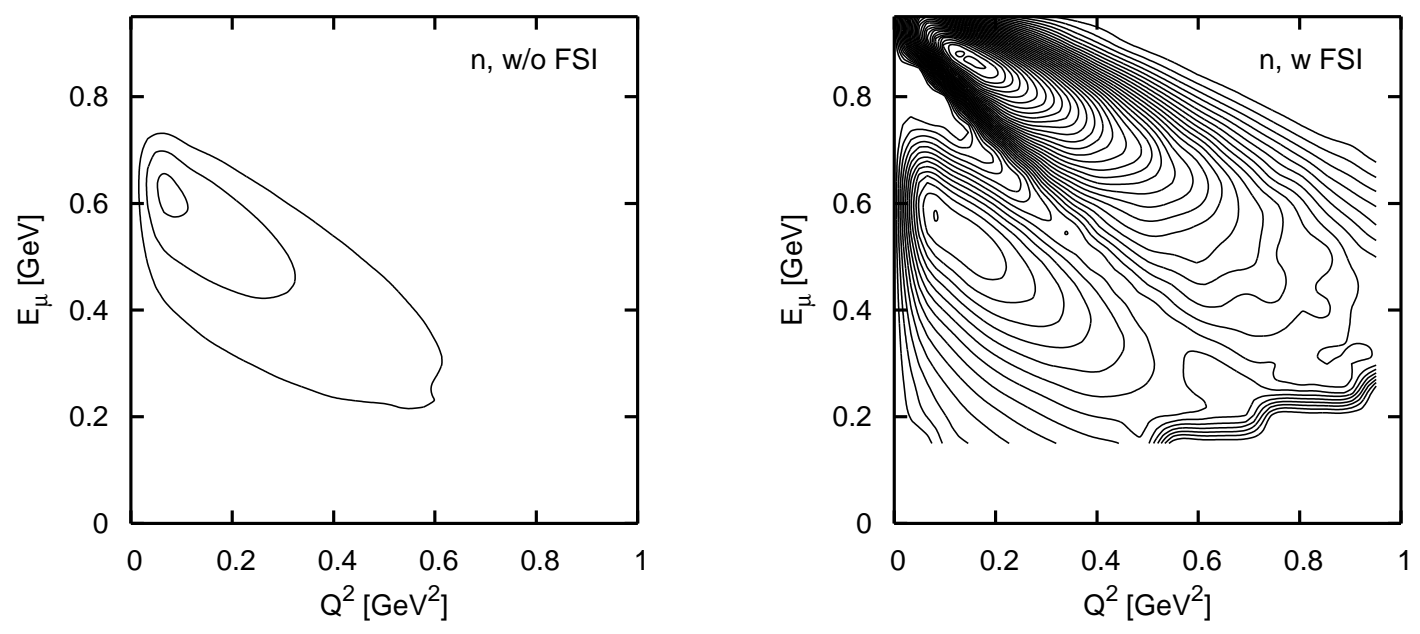

Figure 4. Double differential cross section $d \sigma / d Q^{2} d E_{\mu}$ for neutron knockout on ${ }^{56} F e$ at $E_{\nu}=1 \mathrm{GeV}$. Left: without FSI, right: with FSI (from [23].

Details of the $\pi N$ interactions can be seen in Fig. 5 that shows the ratios of the energy-differential pion production cross sections with and without FSI. Besides an overall suppression to a level of $R \approx 0.4$ for kinetic energies beyond about $200 \mathrm{MeV}$ caused 
by pion absorption through the $\Delta$ resonance the ratio shows a strong increase towards smaller energies that reflects the slowing down of pions through rescattering. The drop at very small energies is then caused by, at this low energy increasingly important, manybody pion absorption. Particularly interesting is that at low energies the $\pi^{0}$ curve rises significantly higher than the $\pi^{+}$ratio and even goes well above 1 . This is due to a coupled channel effect in the final state which always tends to go from the stronger channel to the weaker one and in this case converts positively charged pions into neutral ones.

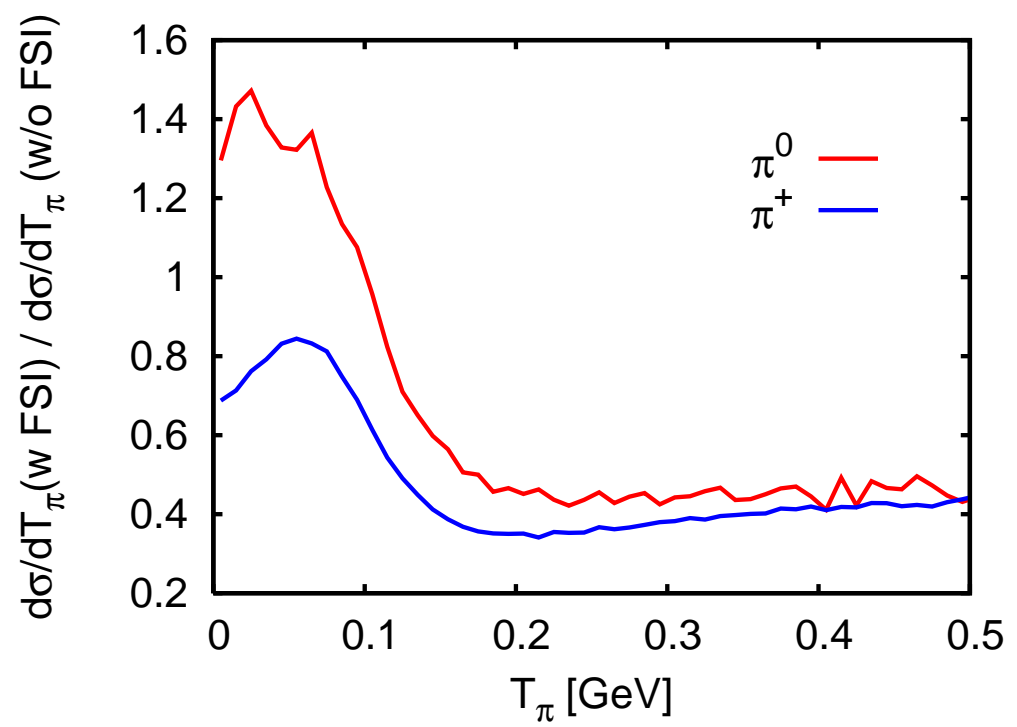

Figure 5. Ratio of energy-differential cross sections for pion production with and without final state interactions for $\pi^{+}$(lower curve) and $\pi^{0}$ (upper curve) for CC interactions of $1 \mathrm{GeV}$ neutrinos on Fe.

\section{Hadron Formation}

A final, interesting example for in-medium effects shows up in the high-energy regime and can be represented as the question how long it takes to produce a particle for example in an electromagnetic interaction process on the nucleon. In the lower-energy regime (a few hundred $\mathrm{MeV}$ incoming energy) this time is often determined by the lifetime of nucleon resonances. For example, pion production on the nucleon predominantly proceeds through the $\Delta$ resonance and the pion's formation time is thus given by the lifetime of the $\Delta$. At higher energies, or invariant masses of the nucleon-photon incoming system above about $2 \mathrm{GeV}$, specific nucleon resonances no longer exist and these highly excited nucleon states are described by string excitations. Phenomenological event generators, that try to incorporate many processes of pQCD, such as FRITIOF or PYTHIA, take care of the decay of such states and also generate the space-time coordinates of such decays [25].

Transport methods have successfully been used to analyze such high-energy hadron production experiments on nuclear targets [26]. In a simplified version of this model 
the prehadrons, just after production through string breaking, experience no interactions within a formation time, $\tau_{f}$, and after that the full hadronic interactions. Since the latter take place only as long as the hadron is still inside the nucleus the nuclear dimension influences the interaction rate. The overall attenuation is then sensitive to the number of collisions inside the medium weighted with the formation time. The energy-loss is then, quite generally, given by

$\Delta E \sim \frac{L}{\lambda} \cdot F(L)$

with $L$ being the distance traveled in the nuclear medium and $\lambda$ the prehadrons mean free path. The first factor is then just the number of collisions. The function $F(L)$ takes into account that an interaction can take place only if the particle has already been formed

$F(L)=\left\{\begin{array}{cc}\frac{L}{\tau_{f}} & L / \tau_{f}<1 \\ 0 & \text { otherwise }\end{array}\right.$

In this simplified model the $L\left(\sim A^{1 / 3}\right)$ dependence is thus already complicated. Indeed, an expansion of (11) in terms of powers of $A^{1 / 3}$ shows a mixture of various powers. The power alone thus does not allow one to distinguish between different models for the energy loss or absorption [26, 27]. This simple model can actually describe the data with a properly adjusted formation time of about $0.5 \mathrm{fm} / \mathrm{c}$ in the restframe of the hadron being formed, if in addition a reduced, but constant prehadronic interaction cross section for leading hadrons is introduced. However, it has been a problem to describe both the HERMES experiments and the EMC attenuation data, taken at considerably higher energies, with the same parameters [28].

Perturbative QCD predicts that the prehadronic cross sections should rise linearly with time [29]. We have recently incorporated such a behavior into our calculations by using for the prehadronic cross sections the expression [30]

$\frac{\sigma_{\mathrm{pre}}}{\sigma_{H}}=\frac{n_{q}}{Q^{2}}\left(1-\frac{t-t_{p}}{t_{f}-t_{p}}\right)+\frac{t-t_{p}}{t_{f}-t_{p}}$.

Here $t_{p}$ and $t_{f}$ denote the production time, i.e. the time at which the quarks inside the hadron are produced through string breaking, and the formation time, respectively. The quantity $n_{q}$ denotes the number of leading quarks, i.e. quarks present in the original hit hadron.

Fig. [6] shows the attenuation as a function of $z=E_{\text {hadron }} / \nu$ demonstrates that such a model indeed gives a very good description of the total hadron attenuation in both the HERMES and the EMC experiments.

\section{Summary}

In this talk various aspects of in-medium effects have been demonstrated. First, the importance of resonance-hole excitations for calculations of in-medium hadronic spectral functions of the $\rho$ and $\omega$ meson has been demonstrated. Since any in-medium signal that involves hadrons in the final states is subject to final state interactions these latter have to be treated with the same accuracy as the in-medium effects themselves. This can 


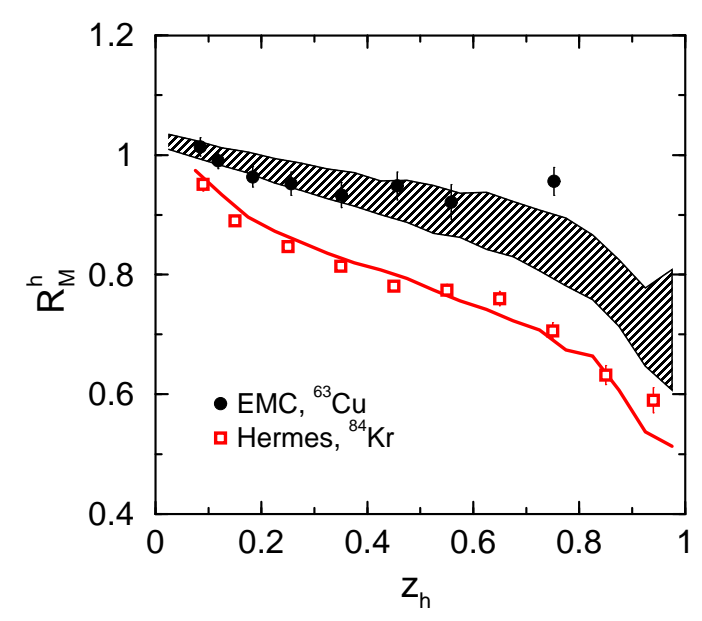

Figure 6. Nuclear attenuation ratio for the HERMES and the EMC experiment. The shaded band for the EMC experiment gives the uncertainty connected with averaging over the beam energies of 100 and $280 \mathrm{GeV}$, in line with the experimental analysis. (from [31]

nowadays be achieved with semiclassical transport methods; usable quantum-mechanical approaches do not exist for the description of inclusive events. Using an example from neutrino-nucleus interactions, charged current neutron knock-out, the overwhelming influcence of final state interactions has been demonstrated. Finally, the potential to determine hadronic formation times in high-energy collisions of photons with nuclei has been pointed out.

\section{REFERENCES}

1. G. Agakichiev et al. [CERES Collaboration], Eur. Phys. J. C 41 (2005) 475 [arXiv:nucl-ex/0506002].

2. J. Adams et al. [STAR Collaboration], Phys. Rev. Lett. 92 (2004) 092301 [arXiv:nuclex/0307023].

3. R. Arnaldi et al. [NA60 Collaboration], Phys. Rev. Lett. 96 (2006) 162302 [arXiv:nuclex/0605007].

4. U. Mosel, Proc. Int. Workshop XXV on Gross Properties of Nuclei and Nuclear Excitations, Hirschegg, Austria, Jan. 13 - 18, 1997, p. 201, GSI Report ISSN 0720-8715; U. Mosel, Progr. Part. Nucl. Phys. 42161 (1999); U. Mosel, Proc. Int. Workshop XXVIII on Gross Properties of Nuclei and Nuclear Excitations, Hirschegg, Austria, Jan. 16-22, 2000, GSI Report ISSN 0720-8715.

5. Ch. Djalali et al, g7 experiment at JLAB

6. D. Trnka et al. [CBELSA/TAPS Collaboration], Phys. Rev. Lett. 94, 192303 (2005) [arXiv:nucl-ex/0504010].

7. T. Falter, J. Lehr, U. Mosel, P. Muehlich and M. Post, Prog. Part. Nucl. Phys. 53 
(2004) 25 [arXiv:nucl-th/0312093].

8. L. Alvarez-Ruso, T. Falter, U. Mosel and P. Muehlich, Prog. Part. Nucl. Phys. 55 (2005) 71 [arXiv:nucl-th/0412084].

9. T. Hatsuda and S. H. Lee, Phys. Rev. C 46, 34 (1992).

10. S. Leupold and U. Mosel, Phys. Rev. C 58, 2939 (1998) [arXiv:nucl-th/9805024].

11. S. Leupold, Nucl. Phys. A 743 (2004) 283 [arXiv:hep-ph/0303020].

12. T.E.O. Ericson and W. Weise, Pions in Nuclei, Clarendon, Oxford, (1988)

13. M. Post and U. Mosel, Nucl. Phys. A 699 (2002) 169 [arXiv:nucl-th/0108017].

14. M. Post, S. Leupold and U. Mosel, Nucl. Phys. A 741 (2004) 81 [arXiv:nuclth/0309085].

15. F. Klingl, N. Kaiser and W. Weise, Nucl. Phys. A 624, 527 (1997) [arXiv:hepph/9704398]; F. Klingl, T. Waas and W. Weise, Nucl. Phys. A 650, 299 (1999) [arXiv:hep-ph/9810312].

16. J. Barth et al, SAPHIR collaboration, Eur. Phys. J. A18, 117 (2003)

17. G. Penner and U. Mosel, Phys. Rev. C 66, 055212 (2002) [arXiv:nucl-th/0207069];

G. Penner and U. Mosel, Phys. Rev. C 66, 055211 (2002) [arXiv:nucl-th/0207066].

18. M. F. M. Lutz, G. Wolf and B. Friman, Nucl. Phys. A 706, 431 (2002) [Erratum-ibid. A 765, 431 (2006)] [arXiv:nucl-th/0112052].

19. V. Shklyar, H. Lenske, U. Mosel and G. Penner, Phys. Rev. C 71, 055206 (2005) [Erratum-ibid. C 72, 019903 (2005)] [arXiv:nucl-th/0412029].

20. P. Muehlich, V. Shklyar, S. Leupold, U. Mosel and M. Post, arXiv:nucl-th/0607061.

21. M. Post and U. Mosel, Nucl. Phys. A 688, 808 (2001) [arXiv:nucl-th/0008040].

22. P. Muhlich, T. Falter and U. Mosel, Eur. Phys. J. A 20 (2004) 499 [arXiv:nuclth/0310067].

23. T. Leitner, L. Alvarez-Ruso and U. Mosel, Phys. Rev. C 73, 065502 (2006) [arXiv:nuclth/0601103].

24. For details see: http://theorie.physik.uni-giessen.de/GiBUU

25. K. Gallmeister and T. Falter, Phys. Lett. B 630, 40 (2005) [arXiv:nucl-th/0502015].

26. T. Falter, W. Cassing, K. Gallmeister and U. Mosel, Phys. Rev. C 70 (2004) 054609 [arXiv:nucl-th/0406023].

27. A. Accardi, D. Grunewald, V. Muccifora and H. J. Pirner, Nucl. Phys. A 761 (2005) 67 [arXiv:hep-ph/0502072].

28. T. Falter, University of Giessen PhD thesis, 2004, http://theorie.physik.unigiessen.de/documents/dissertation/falter_phd.pdf

29. Yu. L. Dokshitzer et aL, Basics of Perturbative QCD (Editions Frontieres, 1991)

30. G. R. Farrar, L. L. Frankfurt, M. I. Strikman and H. Liu, Phys. Rev. Lett. 64 (1990) 2996.

31. K. Gallmeister, U. Mosel, to be published 